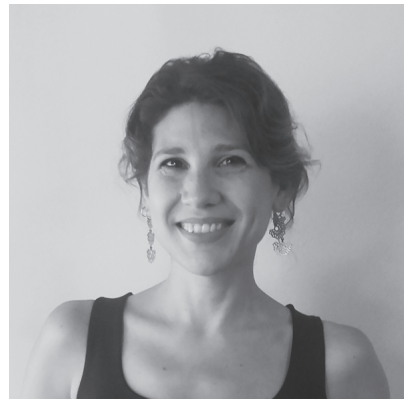

Mariela Baladron

Lic. en Cs. de la Com. Social

Mg. en Industrias Culturales:

Políticas y Gestión. Instituto de

Estudios de América Latina y el

Caribe (IEALC-UBA).

Observatorio Comunicación y

Derechos (DERCOM-FCS-UBA).

mariela.baladron@gmail.com

\title{
El Plan Argentina Conectada: una política de Estado desde la infraestructura de comunicaciones
}

Resumen: El Plan Nacional de Telecomunicaciones "Argentina Conectada" lanzado en 2010, tuvo como eje principal el desarrollo de una Red Federal de Fibra Óptica. Entre sus aspectos novedosos cabe mencionar la inversión pública en la infraestructura de internet, mediante la construcción de una red troncal, un cambio en relación al rol del Estado como inversor y dinamizador del sector desde la empresa estatal de telecomunicaciones (ARSAT); y la articulación con otras iniciativas de democratización del acceso a tecnologías digitales como el Programa Conectar Igualdad y el desarrollo de la Televisión Digital Abierta. Este artículo aborda entre otros aspectos las principales características del diseño e implementación de esta red y el grado de vinculación y participación de actores sociales del sector, en particular del complejo científico tecnológico. Finalmente se analiza su estado actual en el marco de las modificaciones introducidas por el gobierno que asumió en diciembre de 2015.

Palabras clave: acceso a internet; fibras ópticas, REFEFO, políticas públicas.

\section{The "Argentina Conectada" plan: a state policy for communications}

Abstract: The National Telecommunications Plan "Argentina Conectada", launched in 2010, had as its main axis the development of a Federal Fiber Optic Network. [Red Federal de Fibra Óptica -REFEFO]. Among its novel aspects it is worth mentioning the public investment in the internet infrastructure, through the deployment of a backbone network, a change in relation to the role of the State as investor and promoter of the sector through the state telecommunications company (ARSAT); and the articulation with other initiatives to democratize access to digital technologies such as 'Conectar Igualdad' and the development of Open Digital Television. This article addresses, among other aspects, the main characteristics of the design and implementation of this network and the degree of involvement and participation of social actors in the sector, in particular the argentinian science and technology complex. Finally, its current status is analyzed in the framework of the modifications introduced by the government that took office in December 2015. Keywords: internet access, fiber optics, REFEFO, public policies. 
| Ciencia, Tecnología y Política | Año 2 | N² | Enero-Junio 2019 | ISSN 2618-3188 | www.revistas.unlp.edu.ar/CTyP |

\section{O Plano "Argentina Conectada": uma política de Estado para as comunicações}

Resumo: O Plano Nacional de Telecomunicações Argentina Conectada, lançado em 2010, teve como eixo principal o desenvolvimento de uma Rede Federal de Fibra Óptica. Entre os seus aspectos inovadores, vale a pena mencionar o investimento público na infra-estrutura da internet, através da construção de uma rede backbone, uma mudança em relação ao papel do Estado como investidor e dinamizador do setor da empresa estatal de telecomunicações (ARSAT); e a articulação com outras iniciativas para democratizar o acesso a tecnologias digitais, como o Programa Conectar Igualdade e o desenvolvimento da Televisão Digital Aberta. Este artigo aborda, entre outros aspectos, as principais características do projeto e da implementação dessa Rede bem como o grau de envolvimento e participação dos atores sociais do setor, em particular do complexo de científico-tecnológico. Por fim, sua situação atual é analisada no marco das modificações introduzidas pelo governo que tomou posse em dezembro de 2015.

Palavras-chave: acesso à internet; fibras ópticas, REFEFO, políticas públicas

\section{Introducción}

La expansión masiva y comercial de internet desde mediados de la década de 1990 se dio en el contexto político y económico del neoliberalismo, cuya impronta también se observa en las políticas públicas del sector de las telecomunicaciones, con la privatización de las empresas estatales y la implementación de programas pro-mercado que tenían como objetivo conducir a la competencia efectiva. Sin embargo, en la mayoría de los países se generaron mercados oligopólicos o altamente concentrados. Desde finales de la primera década del siglo XXI se observa un cambio de rol de los Estados en el sector de las telecomunicaciones, a través de iniciativas gubernamentales e inversión pública en el despliegue de infraestructura de red, principalmente con la implementación de planes de banda ancha a nivel global y regional. Este enfoque contradice al paradigma predominante en los años previos, que planteaba gobiernos limitados a regular la actividad privada y desarrollar fondos de universalización para compensar las fallas de mercado (Fontanals, 2015b; Galperin, Mariscal y Viecens, 2013).

El análisis de internet en capas o niveles -si bien se trata de una división artificial, ya que estos diferentes niveles interactúan entre sí y sólo en su totalidad constituyen "internet"- permite un análisis más complejo y profundo de las regulaciones jurídicas, la propiedad económica y la dinámica sociológica que opera en cada uno de ellos. Por su parte, Zukerfeld $(2010,2014)$ propone un análisis de cinco niveles o capas: infraestructura, hardware, software, contenidos y red social. En particular, focaliza su trabajo en la infraestructura, ya que "presenta rasgos opuestos a los de las capas superiores (...) [y] exhibe una concentración altísima, está lejos de toda forma de horizontalidad, carece de actores no capitalistas y, efectivamente, el gran público sabe poco o nada de ella" (Zukerfeld, 2010, p.5). Algunos ejemplos son los tendidos submarinos de fibra óptica, las redes troncales continentales y los satélites, propiedad de un pequeño grupo de empresas privadas a nivel global que los controlan.

En este sentido, el Plan Argentina Conectada, a diferencia de otras políticas públicas del sector, destinó la inversión estatal a la capa de infraestructura de internet, un punto crítico por su alta concentración y elevados costos de capital, a través de la construcción de la Red Federal de Fibra Óptica (REFEFO). La misma 
se planteó como una red troncal a cargo del Estado, que permitiera llegar a todo el país con tecnología de última generación, infraestructura multipropósito y capacidad de trasmitir información de diverso tipo, no solo internet, para responder a demandas de alta capacidad en el mediano y largo plazo. El diseño de este tendido no se basó en una estructura heredada sino que respondió a los intereses definidos por el propio Plan (diferente, por ejemplo, a la lógica de mercado del antiguo tendido ferroviario nacional iniciado en 1880), y apuntó a favorecer la interconexión entre prestadores, mayor competencia y horizontalidad en la distribución y acceso a internet (Feider, Galanternik y Meza Ingaramo, 2013).

Este artículo propone un análisis del origen e implementación del Plan Argentina Conectada (2010-2015) -con foco en la REFEFO-, como principal exponente de la política pública de universalización del acceso a internet, su reconfiguración desde diciembre de 2015 y algunas reflexiones en torno a su continuidad.

\section{La creación y características del Plan Argentina Conectada}

En octubre de 2010, a través del Decreto 1552, el Poder Ejecutivo creó el Plan Nacional de Telecomunicaciones "Argentina Conectada" con la definición de siete ejes estratégicos en relación a la inclusión digital, cuyo objetivo principal era el desarrollo, implementación y operación de una Red Federal de Fibra Óptica (REFEFO), que llevaría adelante la empresa estatal ARSAT, con financiamiento del presupuesto nacional. Para diseñar y coordinar su implementación, se creó la Comisión de Planificación y Coordinación Estratégica, instancia institucional dependiente del Ministerio de Planificación Federal, Inversión Pública y Servicios.

El Plan proponía articular con otras iniciativas, destinadas a reducir la brecha digital, impulsadas en el mismo periodo. Estas eran el desarrollo de la Televisión Digital Abierta (TDA) y el Programa Conectar Igualdad. com.ar (PCI). De esta forma se focalizaba en los distintos aspectos del acceso a internet: la construcción de infraestructura de conectividad a través de la REFEFO; el acceso a dispositivos, servicios y aplicaciones con las netbooks de Conectar Igualdad, destinadas a estudiantes y docentes de establecimientos públicos; los set-top-box del Plan Mi TV Digital -decodificadores para recibir la señal digital en los televisores- para poblaciones en situación de vulnerabilidad; y el acompañamiento en el desarrollo de capacidades, uso y apropiación de tecnologías con los Núcleos de Acceso al Conocimiento (NAC) y el trabajo con las netbooks en escuelas.

El lanzamiento de Argentina Conectada se dio en un contexto regional y global caracterizado por el auge de los planes de banda ancha creados con el fin de incrementar la cobertura de redes en los territorios, el uso de internet por sus habitantes y la mejora en la velocidad y calidad de los servicios (Fontanals, 2015b). Algunas posibles explicaciones de la expansión de estos planes en América Latina incluyen el "giro a la izquierda" de los gobiernos de la región a comienzos del siglo XXI y la reorientación de su agenda política hacia una mayor intervención estatal; el rechazo de la opinión pública al proceso de privatización de las empresas de servicios públicos y los limitados resultados en términos sociales; y el desarrollo de planes similares en otros países del mundo, apoyados por organismos internacionales como la Organización para la Cooperación y el Desarrollo Económicos (OCDE), la Comisión Económica para América Latina y el Caribe (CEPAL) y la Comisión de Banda Ancha de UNESCO y UIT (Galperín, Mariscal y Viecens, 2013).

En otros países de la región también se crearon planes de conectividad e infraestructura durante este pe- 
riodo, aunque con distintas características y nivel de inversión pública: mientras que Brasil y Argentina definieron que los activos, operación y ejecución del Plan quedara en manos del Estado, otros países como Colombia, México y Chile siguieron el modelo de asociación público-privada, que implica una menor inversión inicial del erario público a cambio de que la red pase al operador privado luego de un plazo de tiempo acordado (Galperin, Mariscal y Viecens, 2013). Desde la Unión de Naciones Suramericanas (Unasur) se anunció en 2012 el proyecto de crear un "Anillo Óptico Suramericano" para garantizar la interconexión entre los países que estaban realizando estos tendidos en sus territorios y la construcción de cables submarinos para conectar la región con África y Europa. La mayor parte del tráfico de internet de Sudamérica pasa a través de los Estados Unidos, por lo tanto los objetivos de esta iniciativa estaban orientados a proteger la soberanía, otorgar mayor redundancia y descentralización a las redes y reducir los costos de conectividad. Cabe mencionar que durante esos años tomaron estado público las actividades de ciberespionaje ilegal y vigilancia mundial estadounidense a través de la filtración de documentos oficiales por parte de Wikileaks en 2010 y las denuncias de Edward Snowden, ex empleado informático de la Agencia Nacional de Seguridad de ese país en 2013, que incluyeron a gobiernos y presidentes latinoamericanos entre sus objetivos.

En relación al proceso político nacional en el que se enmarcó esta iniciativa y los principales actores locales involucrados (funcionarios públicos, representantes del Sindicato de las Telecomunicaciones FOETRA y de las empresas licenciatarias del servicio básico telefónico -Telecom y Telefónica-), hay distintas versiones sobre la interacción y rol de cada uno en la definición y surgimiento del Plan. Por su parte, Telecom y Telefónica propusieron que el Estado Nacional desarrollara $18.000 \mathrm{~km}$ de infraestructura complementaria a la de sus tendidos, para mejorar sus servicios. Una vez concretadas las obras, estas empresas arrendarían esas re-

Red troncal nacional a construir estimada

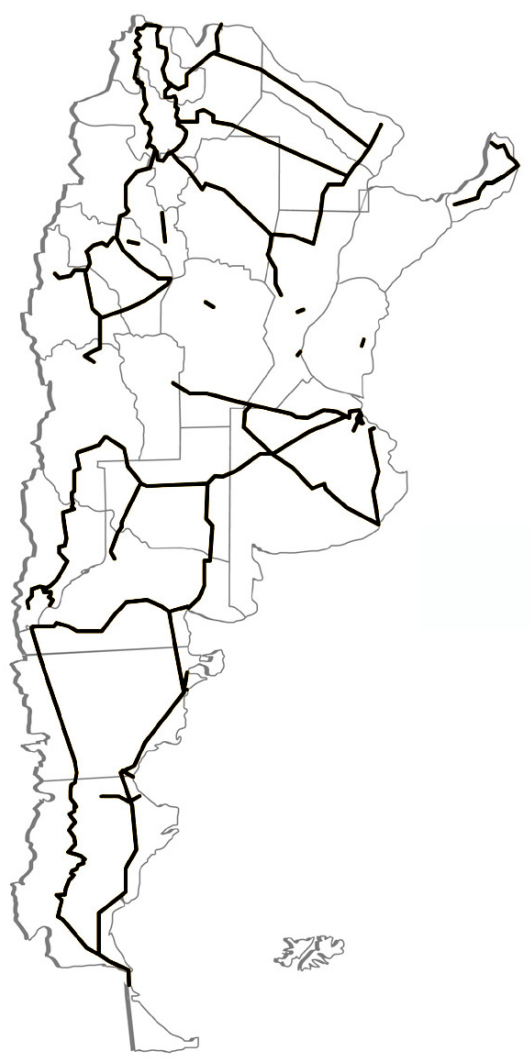

Estimación de las Redes provinciales

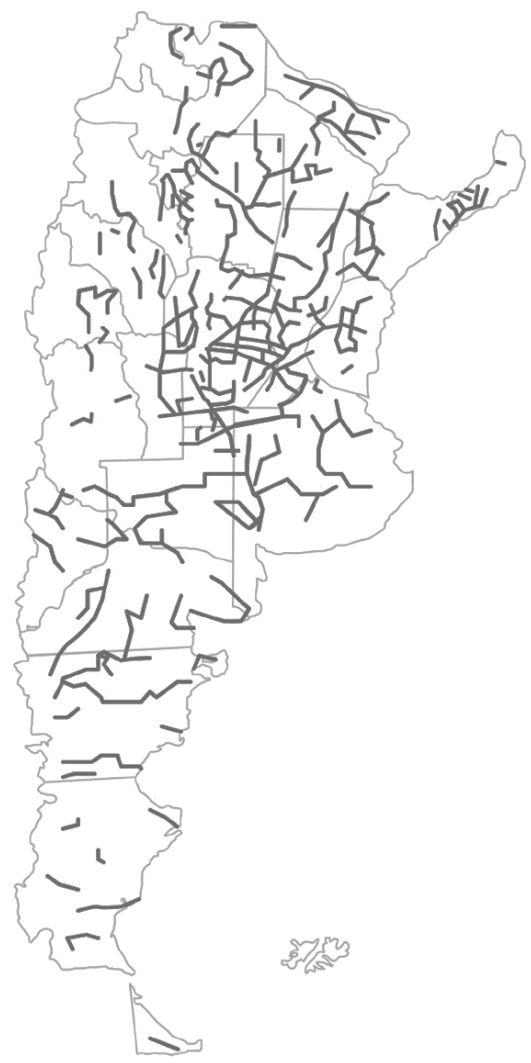

Red Federal de Fibra Óptica 2015

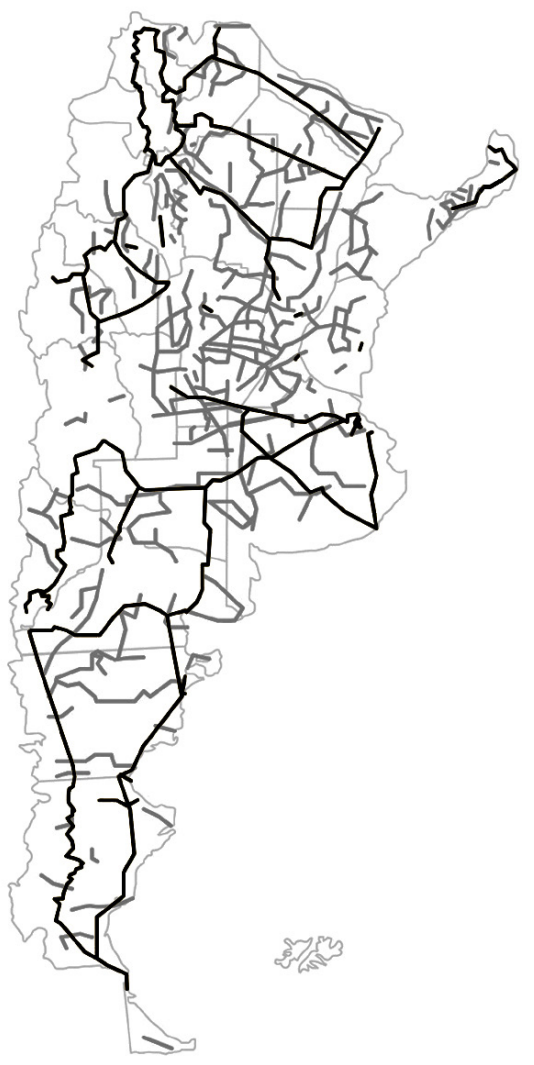

Figura 1. Proyección del tendido troncal y provincial 
des al Estado, que de esta forma recuperaría la inversión, pero sin participar del mercado. Sin embargo, esta propuesta no prosperó porque el objetivo del Plan fue desplegar y operar una red propia de $58.000 \mathrm{~km}$, que articulara la estructura productiva y organizacional del país y firmar contratos de intercambio de infraestructura con estas empresas. Más allá de las implicancias que en términos de soberanía política y tecnológica esta decisión conllevaba, la magnitud de la inversión de capital por parte del Estado en la REFEFO puso de manifiesto una vez más el concepto de que el desarrollo tecnológico y la innovación tienen lugar en el marco de políticas públicas con financiamiento estatal, ya sea con inversiones directas o por investigación y desarrollo aplicado, y no por inversiones de las empresas privadas (Mazzucato, 2015).

El contexto político también pudo haber influido en la decisión de construir la REFEFO. Algunos autores (Krakowiak, 2018) sostienen que un factor desencadenante a nivel local fue el conflicto del gobierno con el Grupo Clarín que prestaba los servicios de acceso a internet por medio de Fibertel. Esta empresa tenía cada vez mayor penetración en el mercado (en 2010 se estimaba en 24,8\% del país) y el gobierno buscó crear ofertas alternativas, ante la posible caducidad de la licencia de Fibertel, promoviendo el desarrollo de PyMES y cooperativas en el marco de la REFEFO.

\subsection{El diseño y las metas de Argentina Conectada}

Con respecto al diagnóstico realizado para la definición del Plan, se consideró la desigualdad geográfica en el acceso de la población al servicio (tanto por razones de disponibilidad como de asequibilidad). Por ejemplo, a fines de 2010, la Ciudad Autónoma de Buenos Aires y la provincia de Buenos Aires reunían el 63\% de los accesos residenciales a internet del país, aunque en estas dos jurisdicciones vivía sólo el 36,1\% de todos los habitantes (INDEC, 2011).

En relación a la concentración del mercado mayorista de internet, se evaluó si la marcada disparidad del precio del "mega" (el valor promedio del Mbps de "Tránsito IP") en distintos puntos del país respondía a razones económicas o técnicas y la conclusión fue que era consecuencia de "cuestiones monopólicas locales" (Jaffrot, comunicación personal)'. Esto se debe a que los operadores más grandes cuentan con sus propios tendidos troncales y realizan acuerdos de interconexión sin costo entre pares o peering, mientras que los ISP puros (acrónimo de Internet Service Providers -proveedores de acceso a internet) deben contratar -según tengan red propia desplegada o no- interconexión, tránsito y última milla² (o alguno de estos servicios), a las empresas integradas verticalmente.

Frente a este contexto, la Comisión de Planificación y Coordinación Estratégica reunió información sobre los tendidos de infraestructura existentes (Fibertel no colaboró con esos datos y las demás empresas lo hicieron parcialmente), y luego definió las trazas de la REFEFO para complementar las zonas que no contaban con infraestructura desarrollada por el sector privado. La REFEFO contemplaba la construcción de puntos de acceso a la red (NAP -acrónimo de Network Access Point) a nivel nacional y provincial, el despliegue de $22.000 \mathrm{~km}$ de fibra óptica de la red troncal y otros $25.000 \mathrm{~km}$ de redes provinciales y la iluminación de

\footnotetext{
${ }^{1}$ Entrevista realizada el 14 de diciembre de 2016 a Emmanuel Jaffrot, secretario Técnico y Académico de la Comisión de Planificación y Coordinación Estratégica del Plan entre 2011 y 2015.

2 Es la red que conecta a los usuarios finales (pueden ser residenciales o corporativos) a la red de las operadoras de telecomunicaciones. Por lo tanto, la REFEFO sería la red mayorista a la que debían conectarse las redes de otras empresas (ISP) para dar servicio a sus usuarios.
} 
tramos de fibra oscura ${ }^{3}$ adquiridos a otras empresas. La REFEFO se planteó como red troncal mayorista a empresas de telecomunicaciones, cooperativas y PyMES y de banda ancha minorista al segmento corporativo y de gobierno, con el objetivo de dinamizar el mercado, aumentar la competencia y bajar los costos de los servicios de conectividad.

Las metas del Plan Argentina Conectada relativas a la REFEFO fueron: brindar cobertura a 1700 localidades, hasta $40 \mathrm{~km}$ de distancia de la red troncal; mejorar la calidad de las conexiones de banda ancha fija, estableciendo 10 Mbps como piso tecnológico de calidad para las nuevas redes; y ampliar la conectividad de los organismos gubernamentales en los ámbitos nacional, provincial y municipal.

\section{Implementación y desarrollo de la REFEFO}

La empresa estatal ARSAT fue creada en 2006 (Ley 26092) para el diseño, construcción, operación y explotación de satélites de telecomunicaciones en el país. A partir de 2010, expandió su alcance al desarrollo y prestación de servicios de otras tecnologías de comunicaciones como la infraestructura de la Plataforma Nacional de Televisión Digital Terrestre (Decreto 364/2010), la REFEFO y el Centro Nacional de Datos del Plan Argentina Conectada. Entre 2011 y 2015, si bien continuó las misma orientación política en el gobierno nacional con la reelección de Cristina Fernández de Kirchner en 2011, las autoridades de ARSAT y de la Secretaría de Comunicaciones fueron modificadas en 2013, lo que coincidió con un cambio en el desarrollo de la Red Federal de Fibra Óptica, que se había iniciado en 2011.

Con fines analíticos, el desarrollo de la REFEFO podría dividirse en dos etapas: la primera tuvo lugar entre 2011 y 2013, bajo la influencia directa del Ministerio de Planificación (titular del 98\% de las acciones de ARSAT y bajo cuya órbita estaba el Plan Argentina Conectada), focalizada en la inversión y despliegue de infraestructura. La segunda etapa, de 2013 a 2015, tuvo mayor hegemonía en la toma de decisiones de otro sector político dentro del partido gobernante (Frente Para la Victoria -FPV-), la organización "La Cámpora", que resolvió como principal objetivo poner en servicio la REFEFO e implementar un plan de negocios con mayor énfasis en el desarrollo comercial.

En el marco de esta redefinición de la REFEFO, durante la segunda etapa se resolvió administrar y poner en servicio $35000 \mathrm{~km}$ de tendidos y dejar en suspenso la proyección original de $58000 \mathrm{~km}$. A diciembre de 2015 se habían completado 29500 km de la REFEFO en cuanto a obra civil concluida, 15700 kilómetros de red iluminada y 14000 con capacidad de dar servicio, aunque la nueva gestión de ARSAT, designada con el cambio de gobierno nacional asumido por Macri en diciembre de 2015, sólo reconoció públicamente 6800 km en servicio (Baladron, 2018; Krakowiak, 2018). Si bien no se cumplió con el cronograma y metas propuestos, cabe mencionar que este despliegue, en comparación con las inversiones del sector privado, fue inédito en cuanto a la extensión y plazos de desarrollo.

\footnotetext{
${ }^{3}$ En el ámbito de las telecomunicaciones, la fibra oscura refiere a los tendidos de fibra óptica desplegados por un operador pero que no están en uso. En general, por los altos costos de la inversión de estas obras, es habitual que se instalen más cables de los que serán utilizados, ya sea para ampliar la capacidad de la red existente en el futuro o para comercializar su uso por parte de terceros. Además de operadores de telecomunicaciones, algunas empresas energéticas o ferroviarias (que también realizan tubos bajo tierra) pueden contar con estos tendidos (por ejemplo, Transener en la Argentina tiene una red nacional de energía eléctrica de alta tensión).
} 
| Ciencia, Tecnología y Política | Año 2 | No2 | Enero-Junio 2019 | ISSN 2618-3188 | www.revistas.unlp.edu.ar/CTyP |

\subsection{Vinculación y participación de actores sociales del sector}

Dada la importancia estratégica del Plan Argentina Conectada, tanto desde un punto de vista tecnológico como económico y social, es llamativo que haya sido prácticamente nula la participación del complejo científico tecnológico en su desarrollo e implementación hasta el momento. Tres años antes del lanzamiento de Argentina Conectada se había creado el Ministerio de Ciencia, Tecnología e Innovación Productiva (MinCyT) ${ }^{4}$ y, sin embargo, no se observa en el Plan Argentina Innovadora 2020 -lanzado en 2013, con los lineamientos para los siguientes 10 años por parte de esa cartera- mención alguna a las comunicaciones en general, las comunicaciones ópticas o la Fotónica, el campo de conocimiento vinculado a estas tecnologías, a pesar de las inversiones que viene realizando el propio Estado en fibra óptica.

Una parte de la comunidad científica ${ }^{5}$ advirtió esta situación y realizó un diagnóstico en términos de demandas y necesidades del país en este campo, junto con propuestas y recomendaciones para la implementación de políticas de investigación, formación de recursos humanos y desarrollo de tecnologías. Las inversiones de ARSAT en equipos ópticos para el desarrollo de la REFEFO requieren de centenas de millones de dólares, lo que implica una clara oportunidad para el desarrollo de empresas, la fabricación nacional, sustitución de importaciones e innovación tecnológica, ya que la oferta disponible en el mercado local corresponde a desarrollos realizados en el exterior o con escaso valor agregado en el país. A su vez, en un contexto de implementación de planes de banda ancha, no existe un proveedor de equipos de comunicaciones ópticas hispanoamericano, lo que representa un potencial para el país y la colaboración regional.

De esta forma, identificaron que las comunicaciones ópticas son un área de vacancia en relación a las necesidades del país y la demanda del propio Estado, tanto en relación a la formación de recursos humanos como para la investigación y desarrollo tecnológico. Por lo tanto, propusieron la articulación con universidades nacionales para un programa de formación en grado y posgrado, y subprogramas para capacitación de técnicos y operadores de campo. Además, solicitaron el apoyo desde los organismos de Ciencia y Técnica para la acreditación de proyectos, convocatorias, equipamiento, desarrollo y creación de laboratorios e instrumentos de financiamiento.

La falta de formación de personal técnico especializado fue reconocida como una de las barreras que enfrentó el Plan Argentina Conectada, particularmente en el desarrollo de las redes provinciales. Las jurisdicciones que lograron un mayor despliegue de tendidos fueron aquellas que ya contaban con empresas tecnológicas o que recibieron un fuerte apoyo político, ya sea desde las gobernaciones o de las secretarías vinculadas al desarrollo de telecomunicaciones y/o tecnología 6 . Otra demanda estuvo relacionada con las PyMES y cooperativas: por un lado, por la demora en la puesta en servicio de la red, lo cual inhibió las inver-

\footnotetext{
${ }^{4}$ Con el Decreto 801/2018 se modificó la estructura ministerial en el país y esta cartera pasó a tener rango de Secretaría. ${ }^{5}$ El 11 septiembre de 2013 en la ciudad de La Plata se realizó la jornada "Ciencia y Tecnología para las Comunicaciones Ópticas en Argentina. Un encuentro entre empresas, industria y sectores académicos para pensar y debatir el rumbo de las Comunicaciones Ópticas en Argentina y articular propuestas de acción conjunta". La misma fue organizada por la Unidad de Vinculación Estratégica de ARSAT, el Comité Territorial de Óptica de Argentina y la División Fotónica y Óptica de la Asociación Física Argentina (AFA). La información de este apartado surge del documento final de esta jornada, disponible en: www.jco2013.blogspot.com.ar.

${ }^{6}$ Las Sociedades Anónimas con Participación Estatal Mayoritaria (SAPEM) tuvieron un rol destacado en el desarrollo de las redes provinciales de la REFEFO, en especial en Chaco, Formosa y La Rioja. Esta evaluación surge de las entrevistas realizadas a Susana Pachecoy, Emmanuel Jaffrot, y Lucas Vicente (Baladron, 2018).
} 
siones en el despliegue de sus propias redes para conectarse con la red mayorista de ARSAT. Por otro lado, se lanzaron líneas de créditos blandos para apoyar sus inversiones, pero este proceso quedó inconcluso.

Por último, cabe mencionar a las redes comunitarias, organizaciones sin fines de lucro que desarrollan su propia red en zonas rurales o de población en situación de vulnerabilidad (en el país se destacan AlterMundi, lanzada en 2012 en las Altas Cumbres de Córdoba, y Atalaya Sur, que comenzó a operar en 2014 en Villa 20 de Ciudad de Buenos Aires). Desde este colectivo se propone, por ejemplo, modificar la perspectiva con la que se concibe el acceso a internet, entendiendo a las personas como la "primera milla" en lugar de la "última milla", invirtiendo la lógica desde arriba hacia abajo o del proveedor al usuario que es el foco de atención predominante sobre internet por parte de las empresas de telecomunicaciones y los Estados (Echániz, 2016). Algunas de las demandas de las redes comunitarias incluyen el acceso al espectro radioeléctrico local y/o regional para los nodos inalámbricos, apoyo con financiamiento del Servicio Universal7 para la capacitación, extensión y mantenimiento de este tipo de redes y la interconexión con la red de ARSAT, por la función social que cumplen.

\section{Diciembre de 2015: cambio de gobierno y nuevo escenario para la REFEFO}

El gobierno de Mauricio Macri llevó adelante profundos cambios en la política pública destinada al sector de las comunicaciones desde el inicio de su mandato en diciembre de 2015. Si bien los objetivos declarados estaban orientados a lograr la convergencia entre el sector audiovisual, de telecomunicaciones y TIC, fomentar la competencia y garantizar la seguridad jurídica para promover inversiones, durante los primeros tres años de su gobierno se verifica en cambio la consolidación de las principales empresas que ya tenían una posición dominante en el mercado argentino y un retroceso en la institucionalidad de la toma de decisiones y la composición del organismo regulador (Califano, 2018; Loreti y De Charras, 2018).

En primer lugar, estas medidas se observan en relación al marco regulatorio, ya que se introdujeron cambios en las leyes del sector (tanto del audiovisual como de las telecomunicaciones) a través de decretos y resoluciones del Poder Ejecutivo, sin consenso ni debate público. Por ejemplo, se redujeron los límites a la concentración económica; se habilitaron nuevas frecuencias del espectro radioeléctrico a los actuales prestadores de telefonía móvil y al Grupo Clarín (a través de la compra de Nextel y otras empresas); se estableció un plazo de 15 años de protección a las redes de nueva generación de última milla de los principales operadores -eximiéndolos de dar acceso a otras empresas más pequeñas y limitando la competencia-; y se aprobó la fusión de Cablevisión y Telecom en 2017, dando origen al mayor jugador del sector infocomunicacional de la región, con niveles de concentración inéditos en los mercados de la TV por cable, la telefonía móvil, la banda ancha y el audiovisual (Decreto 267/2015, Decreto 1340/2016 y Resolución 5644/2017 del Enacom). Además, la infraestructura y los clientes finales que reúne la nueva empresa dan como resultado que sea la única oferente del servicio de acceso a internet en muchas localidades del país.

\footnotetext{
${ }^{7}$ Los Programas de Servicio Universal fueron creados para otorgar subsidios parciales y estimular la inversión en infraestructura del sector privado en regiones rurales y/o con servicios insuficientes y poco rentables. En la Argentina se creó en el 2000 (Decreto 764) y está conformado por los aportes del 1\% de los ingresos de las empresas con licencia TIC. Sin embargo, su implementación tuvo retrasos y poco impacto.
} 
| Ciencia, Tecnología y Política | Año 2 | №2 | Enero-Junio 2019 | ISSN 2618-3188 | www.revistas.unlp.edu.ar/CTyP |

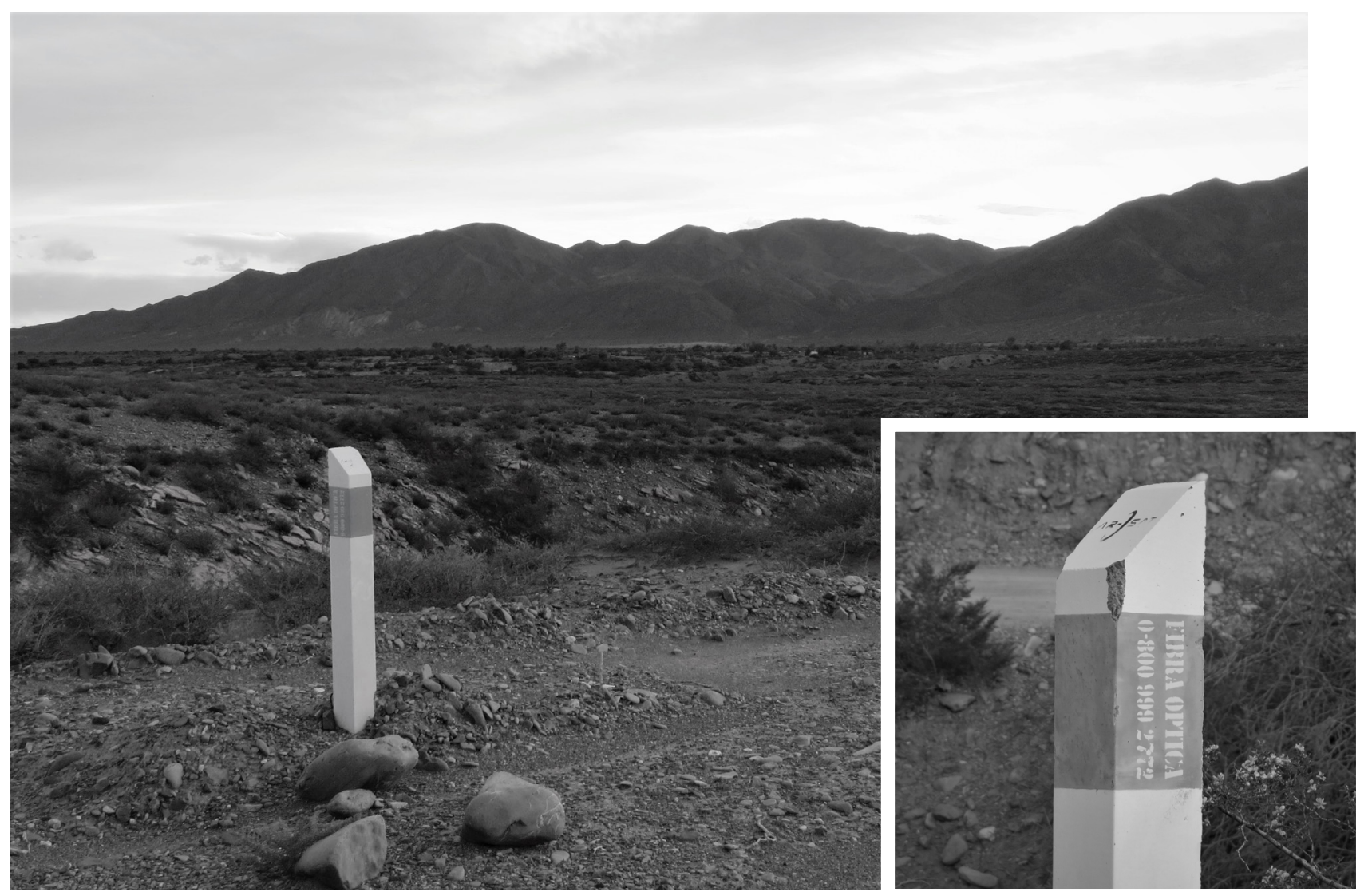

Hitos de hormigón utilizados para la identificación del enlace de fibra óptica y las cámaras de paso y de empalme. Colocados cada $1 \mathrm{Km}$. La foto fue tomada en el km 4551 de la ruta 40 (Provincia de Salta)

En segundo lugar, los planes y programas estatales que se estaban implementando fueron desfinanciados: el desarrollo de la infraestructura de la Televisión Digital Abierta (TDA) quedó estancado y los trabajadores de la unidad encargada de la producción de contenidos fueron despedidos ${ }^{8}$. La entrega de netbooks de Conectar Igualdad fue reduciéndose drásticamente hasta el reemplazo del Programa en 2018 por "Aprender Conectados", que ya no entrega estos dispositivos a estudiantes y docentes. El sector satelital de telecomunicaciones recibió un fuerte impacto con la política de "cielos abiertos", que habilitó el ingreso de operadores extranjeros en detrimento de los servicios prestados por los satélites nacionales ARSAT-1 y ARSAT-2, además de suspender la construcción del ARSAT-3, que ya contaba con contratos firmados y obligaciones establecidas a través de la Ley 27208 de Desarrollo de la Industria Satelital, sancionada en noviembre de 2015. En lo que respecta a la continuidad del Plan Argentina Conectada y la REFEFO, en un primer momento estuvo en consideración la privatización de ARSAT. El gobierno contrató a la consultora McKinsey (vinculada a algunos funcionarios de la nueva gestión) para la elaboración de un plan de negocios en torno a ARSAT. La REFEFO era el activo que suscitaba mayor interés en el sector privado; sin embargo, el lobby cruzado parece ser la razón por la cual se estancó la apertura de la red al capital privado. Finalmente, se definió la continuidad

8El "apagón analógico", es decir, la culminación del proceso de transición de la televisión analógica a la digital, estaba previsto para el 31 de agosto de 2019 (Decreto 1148/2009); sin embargo, ese plazo fue extendido por dos años al cierre de este artículo (Decreto 173/2019). 
de la Red renombrada como Plan Federal de Internet y su anuncio se realizó públicamente el 17 de mayo (día mundial de internet) de 2016 y poco después definió una tarifa plana de ancho de banda mayorista para todo el país.

Si bien se trata de la iniciativa que contó con mayor respaldo y apropiación simbólica por parte del gobierno de Macri, fueron mínimos los recursos que recibió del Tesoro Nacional y su financiamiento procedió casi exclusivamente del Servicio Universal. Por último, sus avances parciales tampoco cumplieron con las metas de la nueva administración: se había establecido el objetivo de brindar banda ancha a 1200 pueblos en los primeros dos años, pero las localidades alcanzadas en ese plazo fueron alrededor de 450, menos de la mitad proyectadas (Krakowiak 2016, 2018). Por lo tanto, aunque la puesta en servicio de la REFEFO siguió avanzando lentamente, no está claro el rol de ARSAT y la vinculación con los actores del sector en el corto y mediano plazo, en un contexto de mayor consolidación económica de los prestadores con posición dominante en el sector.

\section{Conclusiones}

El Plan Argentina Conectada planteó una novedad al proponer un diseño de infraestructura federal para modificar el desequilibrio existente en relación a los tendidos y disponibilidad de conectividad en el territorio nacional, con un rol central por parte del Estado, con la inversión pública y su participación como operador mayorista en el mercado. Si bien no se completaron todos los tramos de la REFEFO y la puesta en servicio se demoró en relación al plazo previsto, se observan importantes avances de las obras de infraestructura desplegadas. Sin embargo, la falta de formación y capacitación de científicos y técnicos y la poca articulación y apoyo a gobiernos locales, PyMES y cooperativas dejó como saldo pendiente los principales objetivos de la Red, en relación a generar mayor competencia y horizontalidad en el acceso a internet. De esta forma, sin una vinculación con la investigación y desarrollo en el campo de las fibras ópticas y los sectores PyME y sin fines de lucro del sector, la REFEFO puede quedar limitada a una herramienta de inclusión digital sin aprovechar su potencial para el desarrollo productivo local y regional con justicia social.

El contexto político en el cual surgió Argentina Conectada en 2010 ha sufrido fuertes cambios en tan sólo un lustro, a nivel internacional y local. Además de las modificaciones ya mencionadas en los planes de gobierno, la política nacional hacia el sector ha introducido fuertes cambios normativos a través de decretos y resoluciones, además de habilitar la fusión entre Cablevisión y Telecom en 2017, lo que implica un nivel de concentración en el sector inédito para el país y la región, lo que afecta sin duda al conjunto de actores que participan en este mercado y marca probablemente un nuevo (o retorno a un viejo) paradigma. En este escenario, un riesgo latente es que la inversión pública en la infraestructura de la REFEFO y su potencial para el desarrollo nacional quede supeditada a los intereses de las principales empresas del sector.

\section{Bibliografía}

BALADRON, M. (2018). El Plan Nacional de Telecomunicaciones Argentina Conectada (2010-2015) en el marco de las políticas públicas de universalización del acceso a internet (tesis de maestría). Universidad 
Nacional de Quilmes (UNQ). Disponible en: https://ridaa.unq.edu.ar/handle/20.500.11807/788 CALIFANO, B. (2018). La regulación de la comunicación durante el primer año de gobierno de Mauricio Macri en la Argentina. Intersecciones en Comunicación 12 (pp.49-74). Facultad de Ciencias Sociales de la Universidad Nacional del Centro de la Provincia de Buenos Aires.

ECHÁNIZ, N. (2016). Redes comunitarias: Internet desde la primera milla. Digital Rights LAC, №30, 8 de abril. Recuperado el 15 de diciembre de 2016 de: https://www.digitalrightslac.net/es/redes-comunitarias-internet-desde-la-primera-milla/

FEIDER, F.; MEZA INGARAMO, C. y GALANTERNIK, V. (2013). Panorama de los planes de infraestructura -telecomunicaciones latinoamericanos. Plan Argentina Conectada. En Revista Hipertextos: Capitalismo, Técnica y Sociedad en debate, 1(0), Buenos Aires (pp. 201-221).

FONTANALS, G. (2015a). "Las telecomunicaciones y la regulación pública. Industrias de red, economías de escala y concentración de mercado". Revista Fibra. Tecnologías de la Comunicación, (3), pp.30-37.

FONTANALS, G. (2015b). "Los planes nacionales de banda ancha en América Latina: la expansión del acceso a internet como política pública". Observatorio Latinoamericano de Regulación, Medios y Convergencia (OBSERVACOM). Recuperado el 20 de marzo de 2016 de: http://www.observacom.org/los-planes-nacionales-de-banda-ancha-en-america-latina-la-expansion-del-acceso-a-internet-como-politica-publica/

GALPERIN, H., MARISCAL, J., y VIECENS, M.F., (2013). "Los planes nacionales de universalización”. En Jordán, V., Galperín, H. y Peres, W. (coords.). Banda ancha en América Latina: más allá de la conectividad (pp. 183.210). Santiago de Chile: CEPAL, DIRSI y @LIS2.

INDEC (Instituto Nacional de Estadisticas y Censos). (2011). Accesos a Internet. Cuarto trimestre de 2010. Buenos Aires.

KRAKOWIAK, F. (2016). "Asoma el plan McKinsey para las comunicaciones”, diario Página/12, 13 de junio.

KRAKOWIAK, F. (2018). "EI plan de internet que saltó la grieta y se convirtió en política de Estado, aunque camina lento", Letra P, 1 de mayo. Disponible en: https://www.letrap.com.ar/nota/2018-5-1-11-44-0-el-plande-internet-que-zafo-de-la-grieta-aunque-camina-lento

LORETI, D. y DE CHARRAS, D. (2018). "La precarización institucional como estrategia" en El Cohete a la Luna, 19 de agosto. Disponible en: https://www.elcohetealaluna.com/la-precarizacion-institucional-como-estrategia/

MAZZUCATO, M. (2015). Innovation Systems: From Fixing Market Failures to Creating Markets. En Mazzucato, M., Cimoli, M., Dosi, G. et al. Which Industrial Policy Does Europe Need? Intereconomics (2015) Mayo 2015, Volumen 50, Número 3, pp 120-155. ZBW y Springer-Verlag Berlin Heidelberg. Recuperado el 10 de octubre de 2016 de https://www.ceps.eu/system/files/IEForum32015.pdf

ZUKERFELD, M. (2010). "De niveles, regulaciones capitalistas y cables submarinos: una introducción a la arquitectura política de Internet". En Revista Virtualis. Sociedad de la Información y del Conocimiento, Número 1, Enero-junio de 2010, Tecnológico de Monterrey, Ciudad de México (pp-5-21).

ZUKERFELD, M. (2014). “Todo lo que usted quiso saber sobre Internet pero nunca se atrevió a googlear”. En Revista Hipertextos, Vol. 1, Número 2, Enero-junio de 2014, Buenos Aires (pp-64-103). 